\title{
NUEVO CONVERTIDOR DC/DC 1-FB-1
}

\author{
Luis Alejandro Gólcher Barguil
}

\section{Resumen}

Se propone un nuevo convertidor DC-DC denominado 1-FB-1. Su topología incorpora un transformador AC para obtener aislamiento galvánico y un número de vueltas, que se utiliza como un segundo grado de libertad en el diseño. Su razón de conversión DC en estado estacionario es igual a uno menos la inversa de la del Convertidor Flyback. Por sus características eléctricas, se puede aplicar en un amplio espectro de aplicaciones horizontales.

Palabras clave: convertidor $\mathrm{dc} / \mathrm{dc}$, convertidor pwm.

\begin{abstract}
A new DC/DC converter is proposed. It is called 1-FB-1. Its electrical topology incorporates a Flyback transformer to obtain DC isolation and a turns ratio. Its DC conversion ratio in steady state is equal to 1 minus the inverse of the Flyback Converter conversion ratio. Due to its electrical characteristics, this converter can be used in a wide range of applications.
\end{abstract}

Key words: dc/dc converter, pwm converter.

Recibido: 16 de noviembre del 2011 • Aprobado: 02 de octubre del 2012

\section{INTRODUCCIÓN}

El convertidor propuesto se denomina 1-FB-1 . Su topología se muestra en la Figura 1.

Si la razón de vueltas se establece en 1:1, el convertidor se asemeja a un Convertidor Boost; observe la Figura 2.

Cuando la razón de vueltas se regresa a 1:n, el Convertidor 1-FB-1 se parece al Convertidor Boost tapped-inductor, exceptuando que el Convertidor 1-FB-1 utiliza un transformador Flyback en lugar de un tapped-inductor. (Refiérase a la Figura 3.) De esta manera, se puede inferir que el convertidor propuesto pertenece a la familia de los convertidores Boost.

\section{ANÁLISIS EN MODO DE CONDUCCIÓN CONTINUO}

El Convertidor 1-FB-1 es un sistema no lineal con dos elementos almacenadores de energía, representados por la inductancia magnetizante del transformador Flyback y por la capacitancia de salida. Para realizar el análisis del convertidor en DC, se utiliza la metodología establecida por Middlebrook y Çuk (1983).

Asumiendo que el convertidor opera en Modo de Conducción Continuo y que las pérdidas son despreciables, se procede a realizar el análisis. Inicialmente se redibuja el convertidor con las variables relevantes, ver Figura 4. 


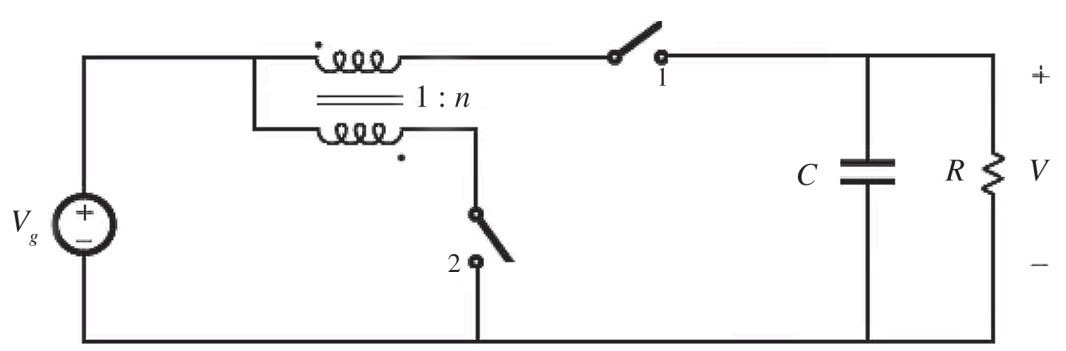

Figura 1. Topología del Convertidor 1-FB-1.

Fuente: Elaboración propia.
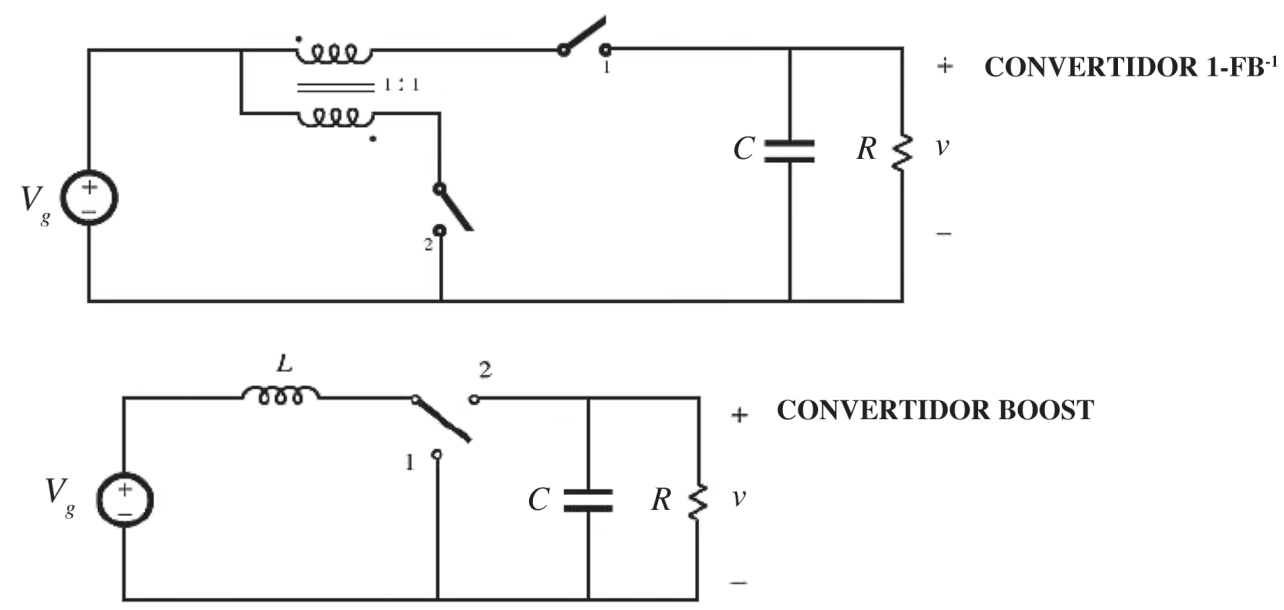

Figura 2. Topología del Convertidor 1-FB-1 en comparación con el Convertidor Boost.

Fuente: Elaboración propia.
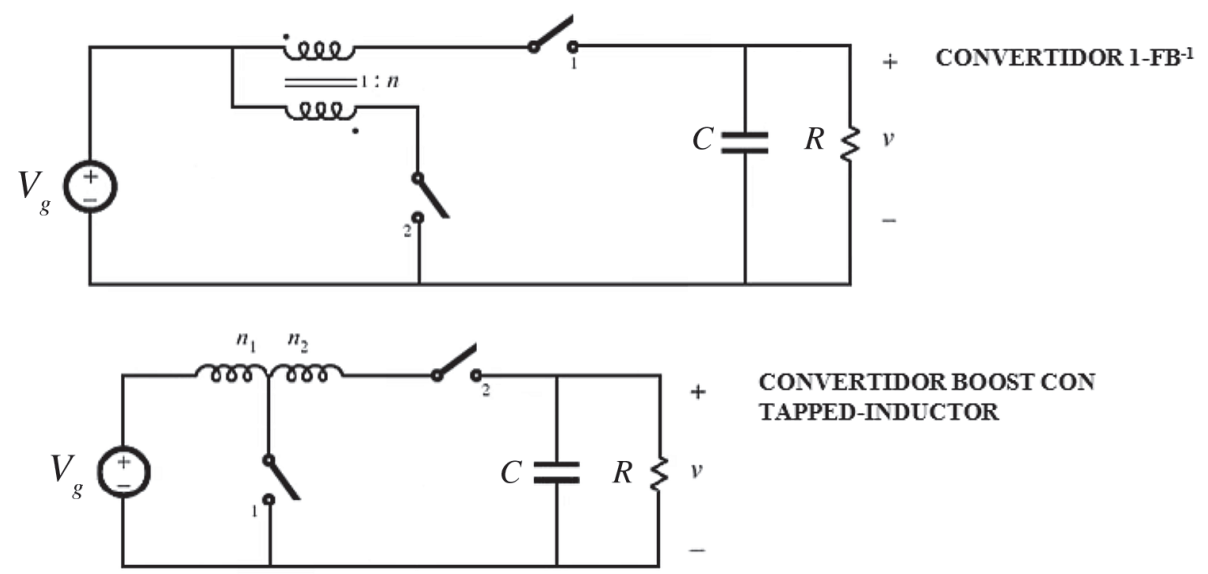

Figura 3. Topología del Convertidor 1-FB-1 en comparación con el Convertidor BoostTapped-Inductor. Fuente: Elaboración propia. 


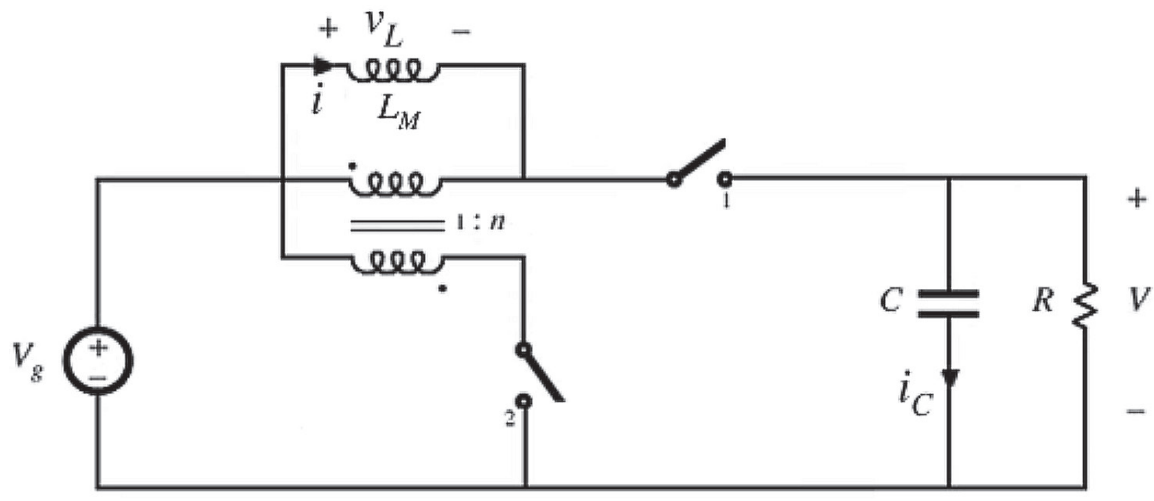

Figura 4. Variables del Convertidor 1-FB-1. Fuente: Elaboración propia.

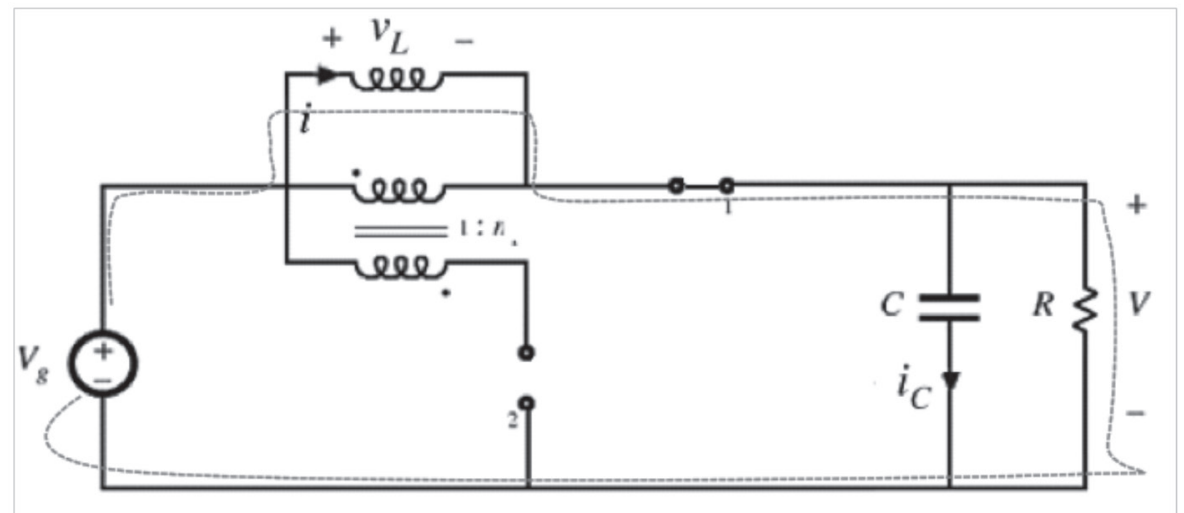

Figura 5. Intervalo I del periodo de conmutación. Fuente: Elaboración propia.

En el primer intervalo del periodo de conmutación, $0<t \leq D T_{s}$, el switch cierra a la posición 1 , y se abre el switch de la posición 2. La topología queda como se muestra en la Figura 5. Las ecuaciones diferenciales de los estados quedan establecidas de la siguiente forma:

$V_{L}=(t) \cong V_{g}-V$

$$
i_{c}(t) \cong I-\frac{V}{R}
$$

Durante el segundo intervalo del periodo de conmutación, $D T_{s}<t \leq T_{s}$, el switch cierra a la posición 2 , y se abre el switch de la posición 1 . La topología queda como se muestra en la Figura 6.
Las ecuaciones diferenciales de los estados quedan establecidas de la siguiente forma:

$$
\begin{aligned}
& v_{L}(t) \cong-\frac{V_{g}}{n} \\
& i_{C}(t) \cong-\frac{V}{R}
\end{aligned}
$$

Aplicando el principio de balance de voltsegundos en estado estacionario:

$$
<v_{L}>=D\left(V_{g}-V\right)+D^{\prime}\left(-\frac{V_{g}}{n}\right)=0, \text { con } D^{\prime} \triangleq 1-D
$$

Donde se desprende que la razón de conversión de tensiones en DC es: 


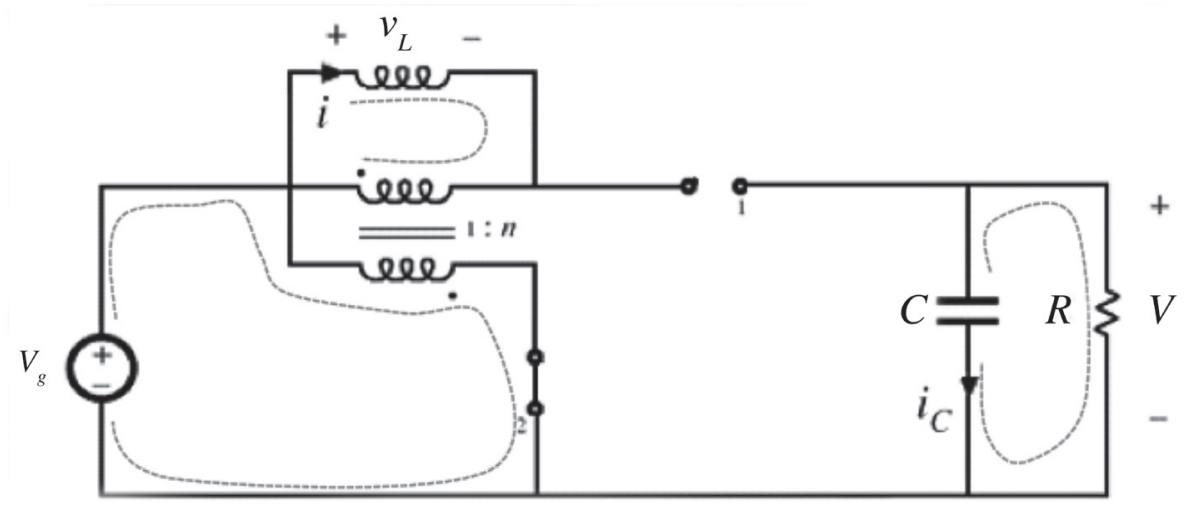

Figura 6. Intervalo II del periodo de conmutación.

Fuente: Elaboración propia.
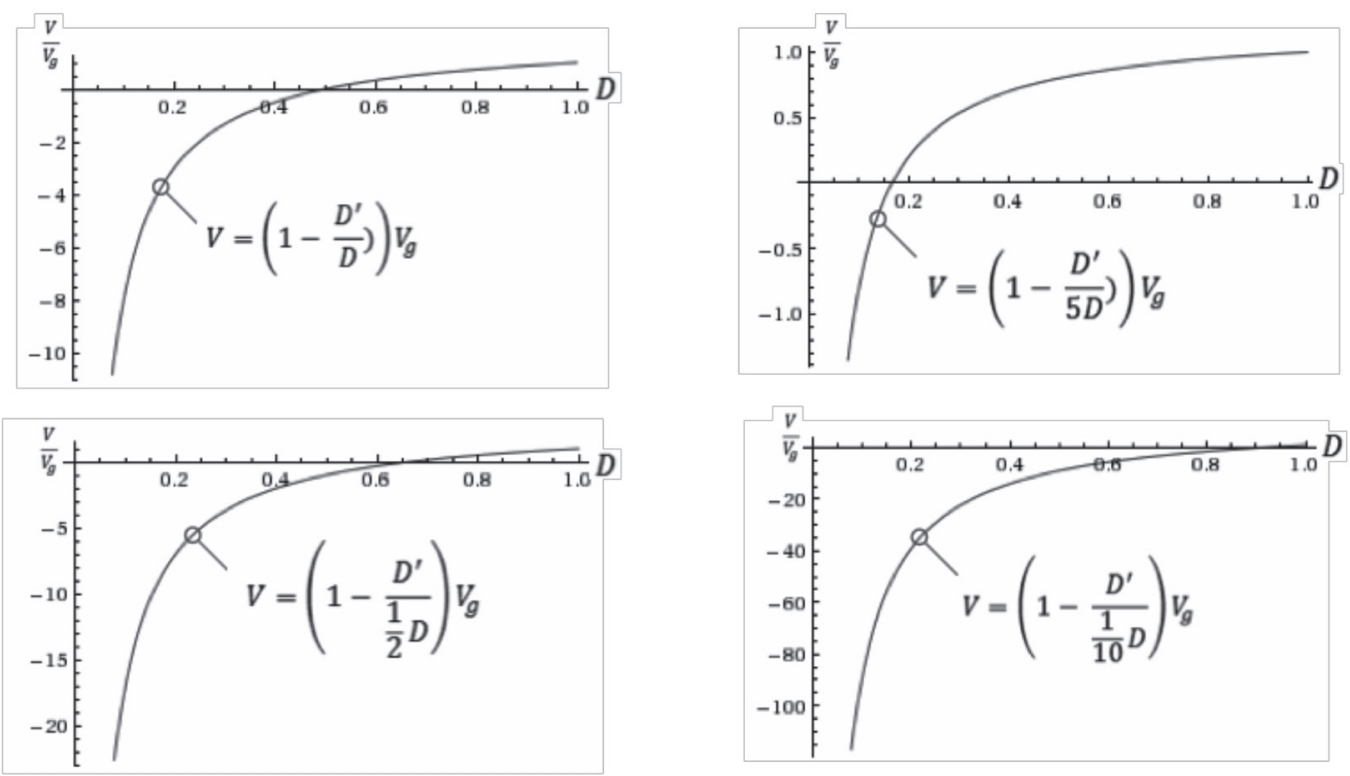

Figura 7. Razones de Conversión de Tensión DC para distintos números de vuelta.

Fuente: Elaboración propia.

$$
V=\left(1-\frac{D^{\prime}}{D n}\right) V_{g}
$$

La Figura 7 muestra el comportamiento de la razón de conversión de voltajes $V / V_{g}$ para distintas razones de vuelta. La razón de conversión de voltaje DC tiende en general a invertir y aumentar la tensión de entrada para $\mathrm{D}$ pequeños; mientras que para $\mathrm{D}$ más cercanos a 1 , la razón de conversión de tensión DC tiende hacia 1.
Por ejemplo para una razón de vueltas 1:1, si se desea disminuir la tensión de entrada, se debe operar el convertidor con D > 1/2; mientras que si se desea aumentar la tensión el voltaje de entrada, se debe operar el convertidor con $\mathrm{D}<1 / 2$.

Aplicando el principio de balance de amperesegundos en estado estacionario:

$$
<i_{C}>=D\left(I-\frac{V}{R}\right)+D^{\prime}\left(-\frac{V}{R}\right)=\mathbf{0}
$$


Donde se desprende que la corriente DC de magnetización es

$$
I=\frac{1}{D}\left(1-\frac{D^{\prime}}{D n}\right) V_{g} / R
$$

Los rizados de la corriente de magnetización y la tensión del capacitor quedan establecidos en las Ecuaciones 9 y 10 respectivamente. $T_{s}$ es el periodo de conmutación.

$$
\begin{gathered}
\Delta i=\frac{D^{\prime} T_{S} V_{g}}{2 n L} \\
\Delta v=\frac{D^{\prime} T_{S} V}{2 R C}
\end{gathered}
$$

El comportamiento de la corriente de magnetización y la tensión del capacitor queda descrito en la Figura 8.

\section{MODELO DC DEL CONVERTIDOR 1-FB-1}

El modelo es un circuito que representa fidedignamente el comportamiento DC del Convertidor $1-\mathrm{FB}^{-1}$. La ventaja del modelo es que no contiene los switches, lo que lo hace muy útil para realizar manipulaciones de circuitos.

El modelo se deriva de las ecuaciones de balance de volt-segundos y ampere-segundos, Ecuaciones (5) y (7) respectivamente. Estas ecuaciones describen el comportamiento del Convertidor $1-\mathrm{FB}^{-1}$ en DC. Se observa que la ecuación de volt-segundos es una malla de Kirchhoff mientras que la ecuación de ampere-segundos es un nodo de Kirchhoff. Cualquier circuito que se reduzca a esta malla y nodo de Kirchhoff es un modelo del convertidor.

Por inspección a partir de las Ecuaciones (5) y (7), el autor propone el modelo de la Figura 9.

Los transformadores con la línea gruesa en el medio representan un elemento transformador DC que funciona igual que un transformador AC excepto que no se pueden construir físicamente.

Este modelo se puede extender para incorporar las pérdidas del convertidor.

\section{DISEÑO DE LOS SWITCHES}

Inicialmente se redibuja el convertidor con las variables relevantes de los switches (Referirse a la Figura 10). El switch que cierra en el primer intervalo se le denomina Switch A; el switch que cierra en el segundo intervalo se le denomina Switch B.

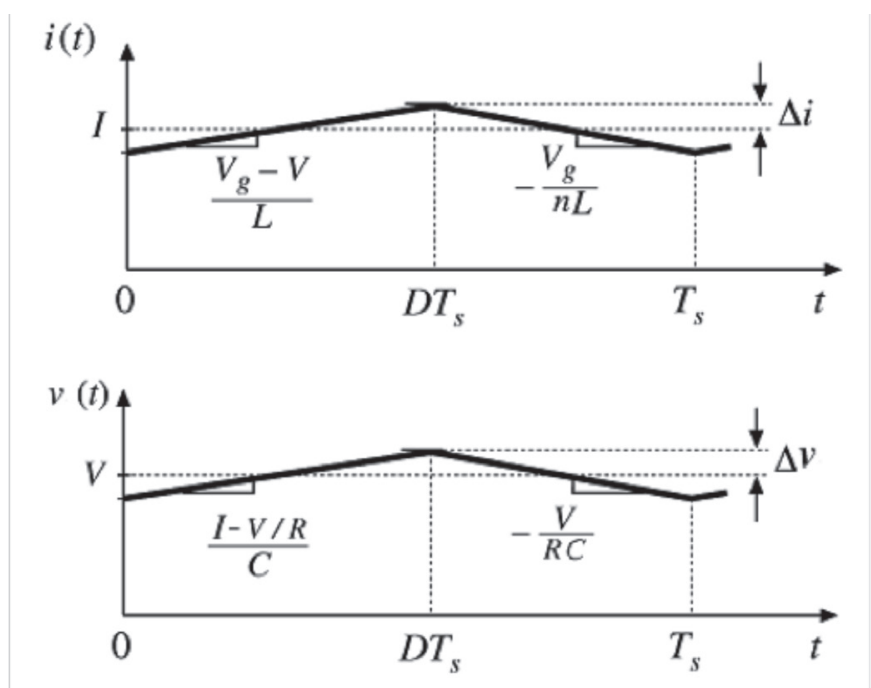

Figura 8. Comportamiento de la corriente de magnetización y voltaje del capacitor en un periodo de conmutación.

Fuente: Elaboración propia. 

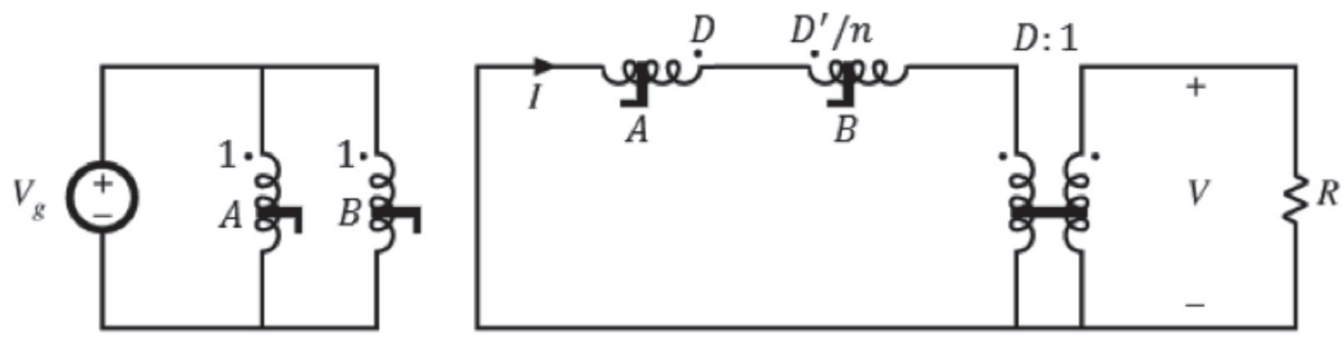

Figura 9. Modelo DC del Convertidor 1-FB-1.

Fuente: Elaboración propia.

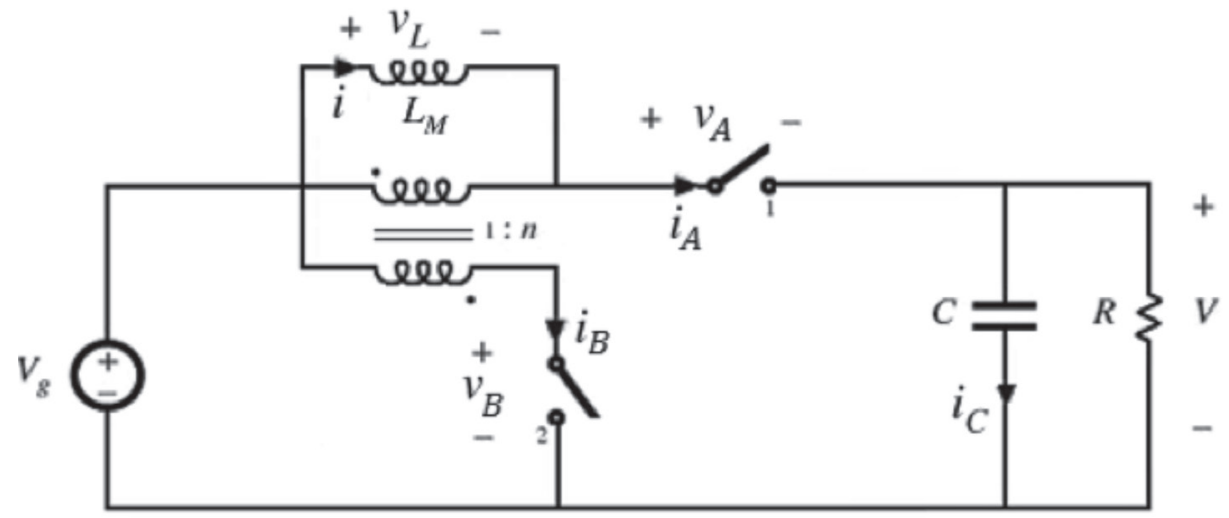

Figura 10. Convertidor $1-\mathrm{FB}^{-1}$ con las variables de los switches.

Fuente: Elaboración propia.

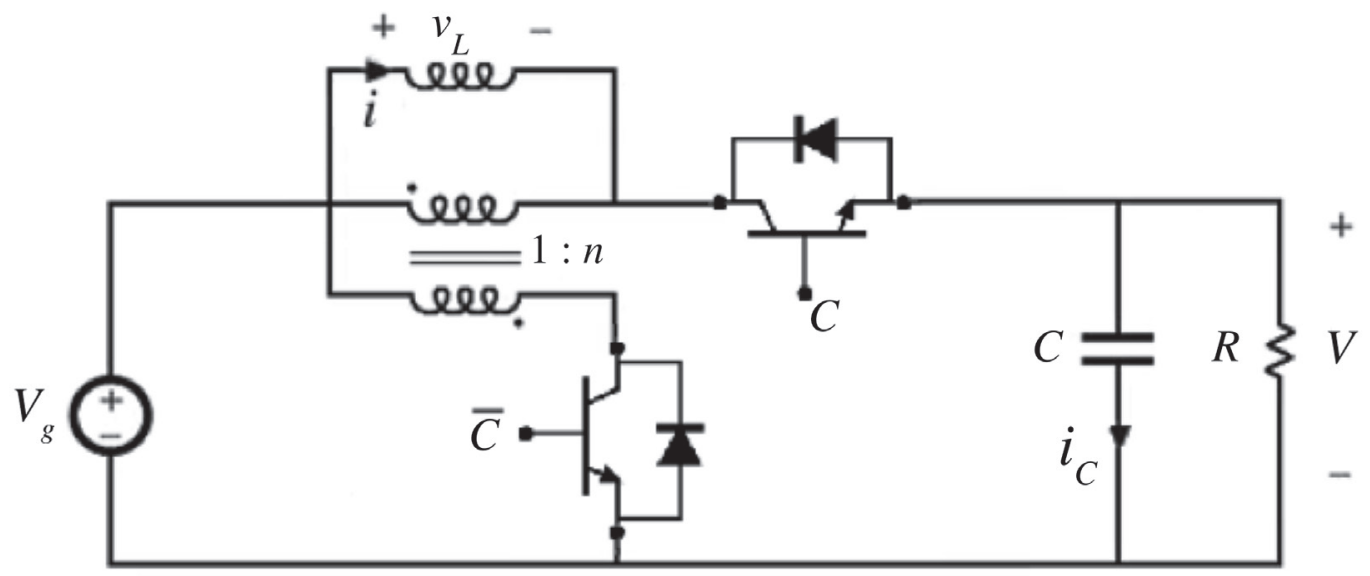

Figura 11. Convertidor $1-\mathrm{FB}^{-1}$ con los switches diseñados.

Fuente: Elaboración propia. 
En el primer intervalo del periodo de conmutación, $0<t \leq D T_{s}$, el Switch A cierra a la posición 1 y se abre el Switch B de la posición 2 . De esta forma:

$$
\begin{aligned}
& i_{A}(t) \cong I \\
& v_{B}(t) \cong V_{g}\left(1+n-1+\frac{D^{\prime}}{n D}\right)=V_{g}\left(n+\frac{D^{\prime}}{n D}\right)
\end{aligned}
$$

Durante el segundo intervalo del periodo de conmutación, $D T_{s}<t \leq T_{s}$, el Switch B cierra a la posición 2, y se abre el Switch A de la posición 1. De esta forma:

$$
\begin{aligned}
& v_{A}(t) \cong V_{g}\left(1+\frac{1}{n}-1+\frac{D^{\prime}}{n D}\right)=V_{g}\left(\frac{1}{n D}\right) \\
& i_{B}(t) \cong-\frac{I}{n}
\end{aligned}
$$

El Switch A debe conducir corriente bidireccional (Ecuación 11) y bloquear tensión positiva (Ecuación 13) por lo que se diseña con un transistor BJT en paralelo con un diodo como se muestra en la Figura 11. El Switch B debe conducir corriente bidireccional (Ecuación 14) y bloquear tensión positiva (Ecuación 12) por lo que se diseña también con un transistor BJT en paralelo con un diodo como se muestra en la Figura 11.

\section{SIMULACIÓN EN MODO DE CONDUCCIÓN CONTINUO}

Dado que el convertidor presenta en su razón de conversión de tensiones un comportamiento inversor y no-inversor, se realizan una simulación para cada caso. Las simulaciones se desarrollan en el ambiente GeckoCircuits.

La primera simulación considera un Convertidor $1-\mathrm{FB}^{-1}$ con $V_{g}=12$ Volts, $D=0.8, L_{M}=100$ $\mu H, R=1$ Ohms, $f_{s}=12 \mathrm{KHz}, n=1$ y $C=470 \mu \mathrm{F}$. De acuerdo a las Ecuaciones (6), (8), (9) y (10), se esperan los siguientes valores en los estados:

$V=\left(1-\frac{D^{\prime}}{D n}\right) V_{g}=\left(1-\frac{0.2}{0.8}\right) 12=9$ Volts

$$
\begin{aligned}
& \mathbf{\Delta} v=\frac{D^{\prime} T_{s} V}{2 R C}=\frac{(0.2)(9)}{2(1)(470 \mu)(60 K)} \cong 32 \text { mVolts } \\
& I=\frac{\frac{\mathbf{1}}{D} V}{R}=\frac{\frac{\mathbf{1}}{\mathbf{0 . 8}} \mathbf{9}}{\mathbf{1}} \cong 11.25 \mathrm{Amps} \\
& \mathbf{\Delta} i=\frac{D^{\prime} T_{s} V_{g}}{2 n L}=\frac{(0.2)(12)}{2(100 u)(60 \mathrm{~K})} \cong 0.2 \mathrm{Amps}
\end{aligned}
$$

Los rizados son considerablemente pequeños con respecto a sus valores DC, por lo que se ratifica que se está operando en Modo de Conducción Continuo. El circuito utilizado en la simulación se muestra en la Figura 12, mientras que el resultado de la simulación se observa en la Figura 13.

Dado que la corriente de la inductancia de magnetización es positiva (Ecuación 17), se requiere únicamente el transistor denominado IGBT.2 para realizar el Switch A y el diodo D.2, para realizar el Switch B.

Se identifica de las formas de onda del diodo y del transistor que el convertidor está operando en Modo de Conducción Continuo. Las pérdidas inherentes a la mayoría de elementos utilizados en el circuito de simulación se minimizaron. La tensión DC del capacitor simulado es de 8.96 Volts, mientras que el teórico esperado es 9 Volts (Ecuación 15). La corriente DC de la inductancia de magnetización simulada es de 11.47Amps, mientras que el teórico esperado es de 11.25 Amps. Ambos valores corroboran la operación teórica del Convertidor 1-FB-1. Las diferencias se deben principalmente a las pérdidas de conmutación, conducción, y cobre en el transformador con las del núcleo.

La segunda simulación considera un Convertidor $1-\mathrm{FB}^{-1}$ diseñado para funcionar con tensión negativa de salida. Los parámetros de diseñado seleccionados son $V_{g}=12$ Volts, $D=0.2$, $L_{M}=100 \mu \mathrm{H}, R=1 \mathrm{Ohms}, f_{s}=12 \mathrm{KHz}, n=1$ y $C=470$ $\mu F$. De acuerdo a las Ecuaciones (6), (8), (9) y (10), se esperan los siguientes valores en los estados:

$$
V=\left(\mathbf{1}-\frac{D^{\prime}}{D n}\right) V_{g}=\left(\mathbf{1}-\frac{0.8}{0.2}\right) 12=-36 \text { Volts }
$$




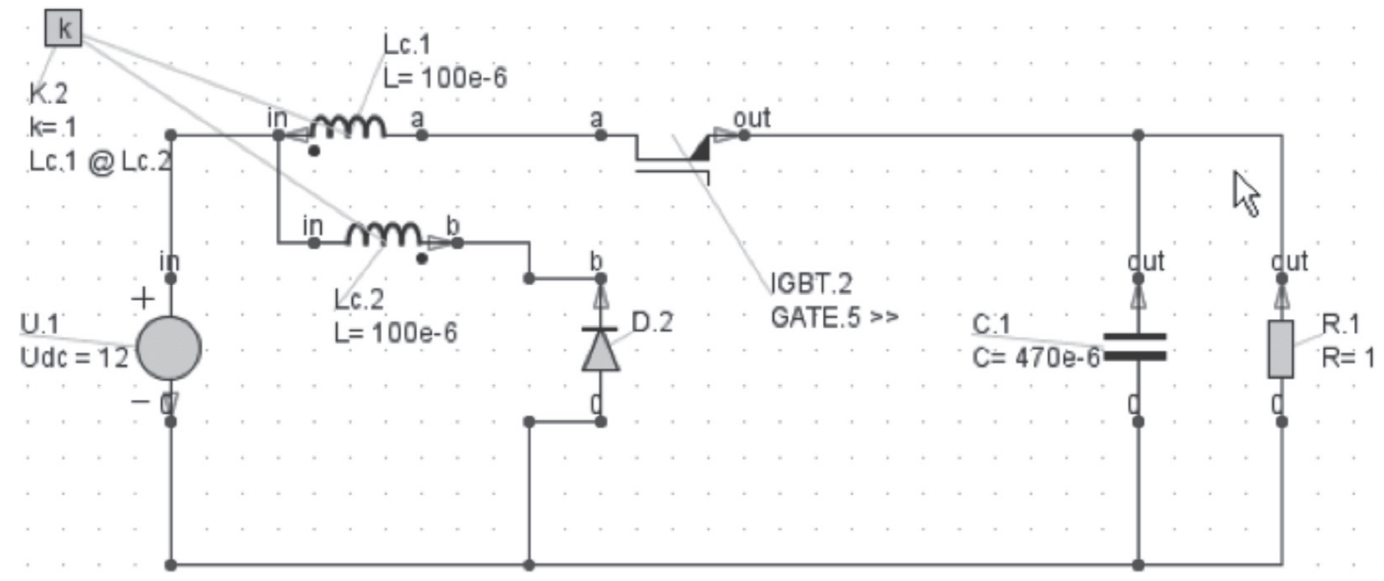

Figura 12. Circuito del Convertidor $1-\mathrm{FB}^{-1}$ simulado. Fuente: Elaboración propia.

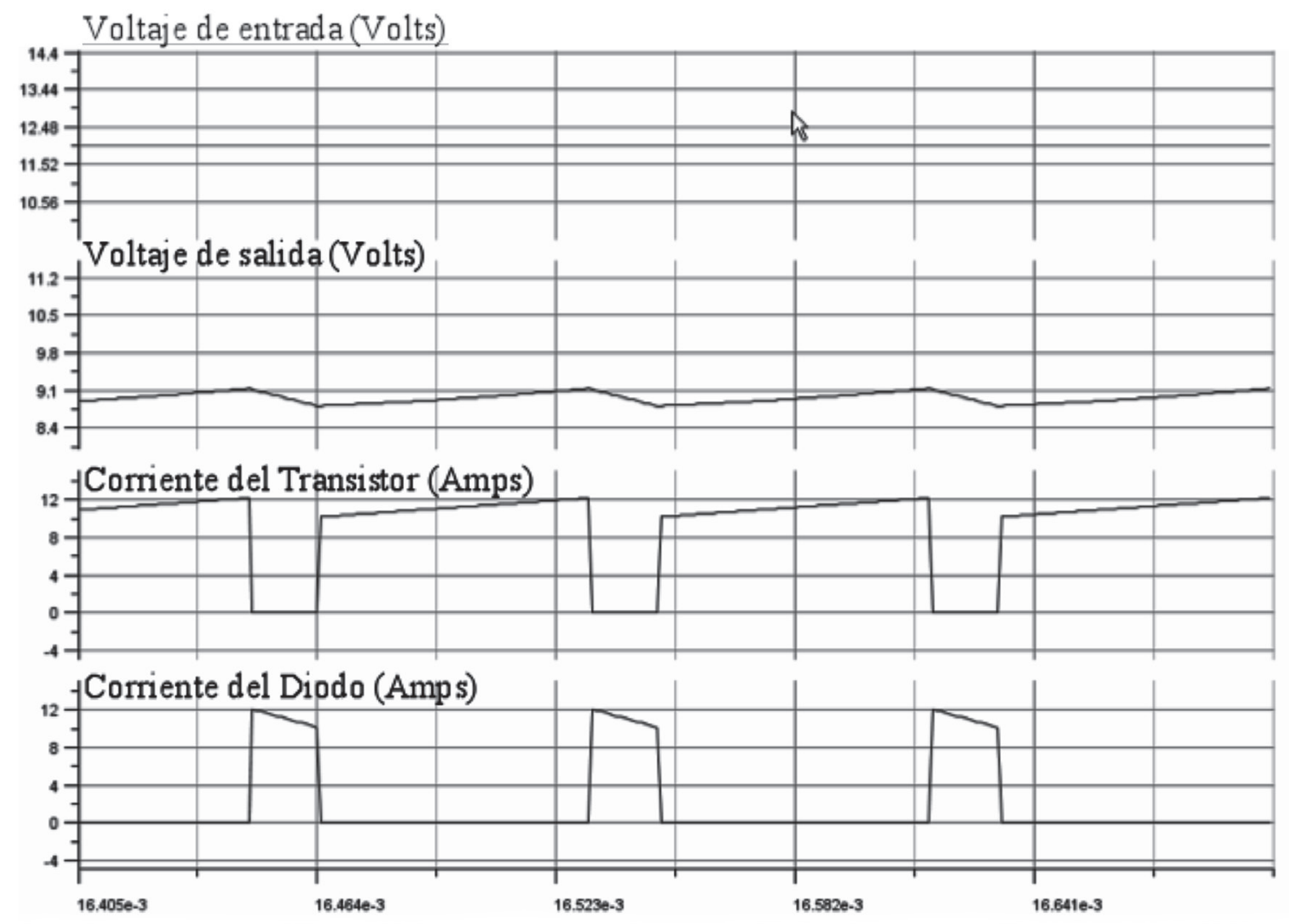

Figura 13. Formas de Onda simuladas del Convertidor 1-FB-1 Fuente: Elaboración propia. 


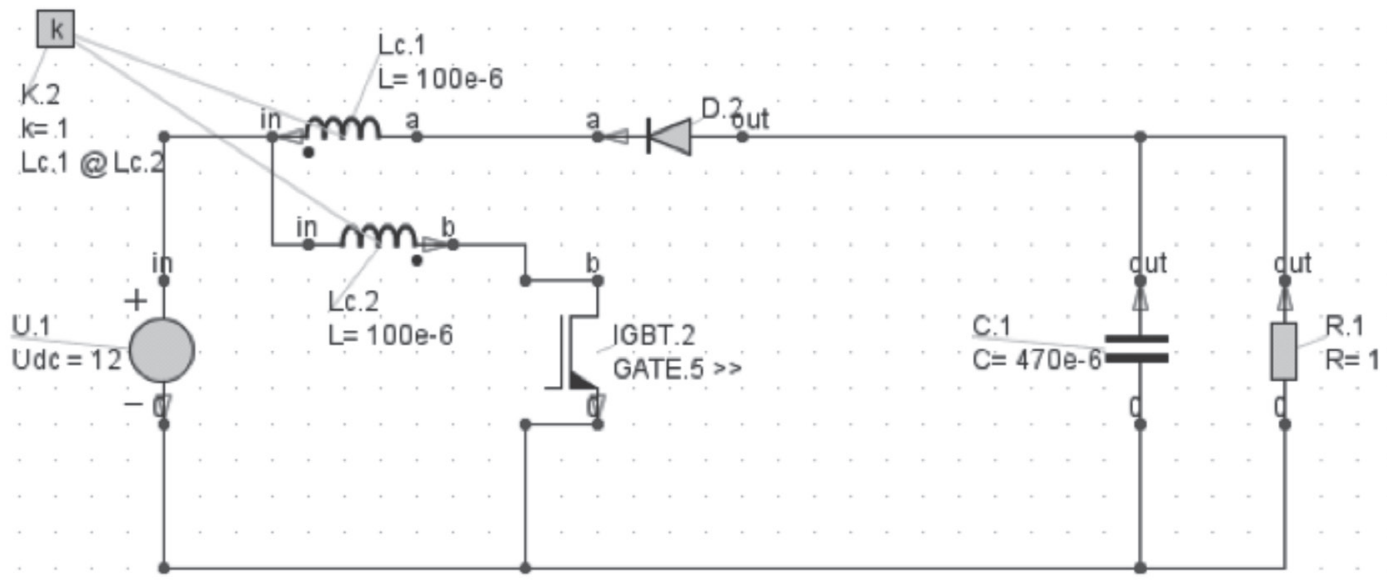

Figura 14. Circuito del Convertidor $1-\mathrm{FB}^{-1}$ simulado. Fuente: Elaboración propia.

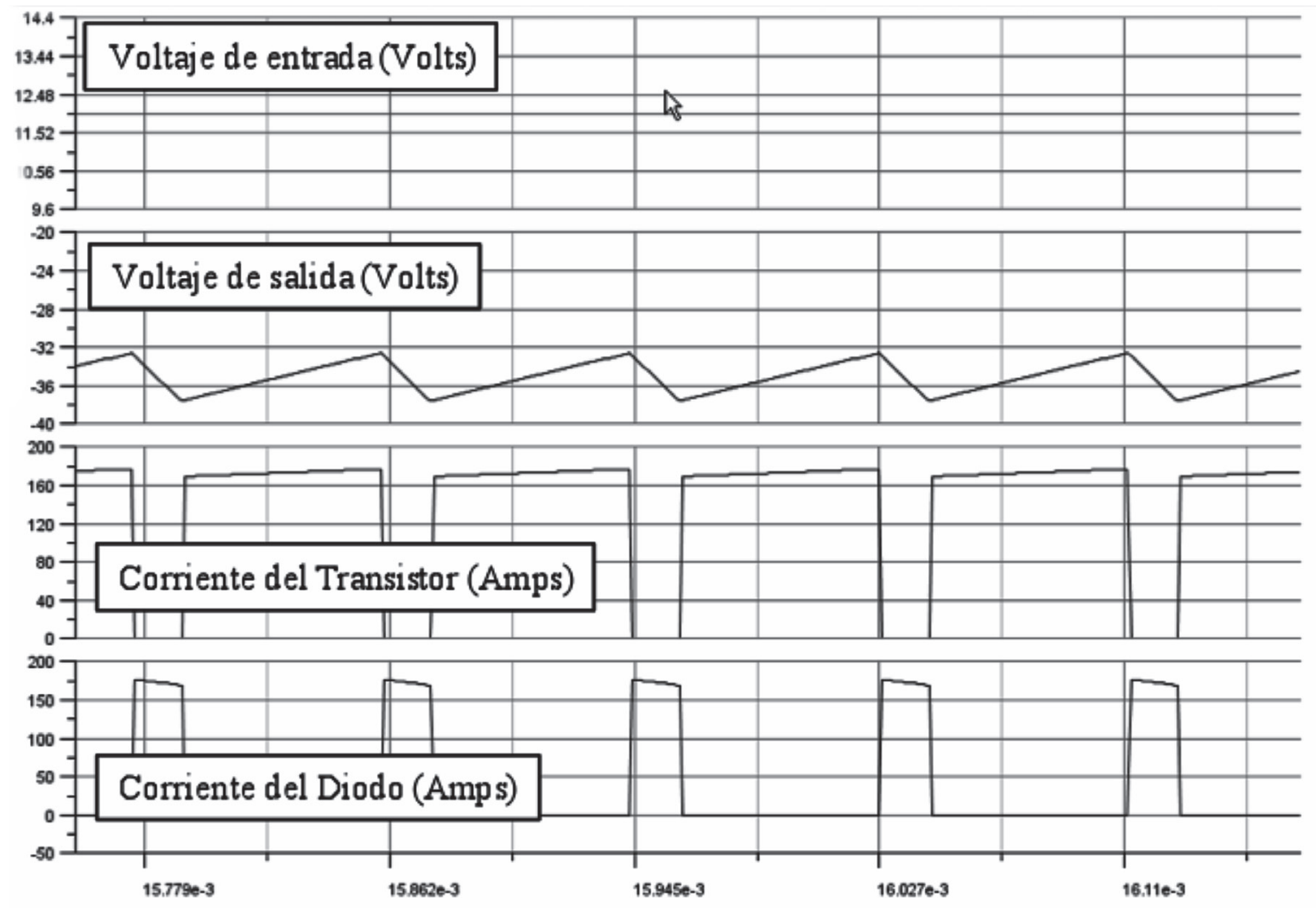

Figura 15. Formas de Onda simuladas del Convertidor 1-FB-1. Fuente: Elaboración propia. 
$\Delta v=\frac{D^{\prime} T_{s} V}{2 R C}=\frac{(0.8)(36)}{2(1)(470 \mu)(60 K)} \cong 0.51$ Volts

$I=\frac{\frac{1}{D} V}{R}=\frac{\frac{1}{0.2}(-36)}{1} \cong-180 \mathrm{Amps}$

$\boldsymbol{\Delta} i=\frac{D^{\prime} T_{s} V_{g}}{2 n L}=\frac{(0.8)(12)}{2(100 u)(60 K)} \cong 51 \mathrm{mAmps}$

Los rizados son considerablemente pequeños con respecto a sus valores DC, por lo que se ratifica que se está operando en Modo de Conducción Continuo. El circuito utilizado en la simulación se muestra en la Figura 14, mientras que el resultado de la simulación se observa en la Figura 15.

Dado que la corriente de la inductancia de magnetización es negativa (Ecuación 21), se requiere únicamente el transistor denominado IGBT.2 para realizar el Switch B y el diodo D.2, para realizar el Switch A.

Se identifica de las formas de onda del diodo y del transistor que el convertidor está operando en Modo de Conducción Continuo. Las pérdidas inherentes a la mayoría de elementos utilizados en el circuito de simulación se minimizaron. La tensión DC del capacitor simulado es de -35.10 Volts, mientras que el teórico esperado es -36 Volts (Ecuación 15). La corriente DC de la inductancia de magnetización simulada es de -174.5Amps, mientras que el teórico esperado es de -180 Amps. Ambos valores y formas de onda corroboran la operación teórica del Convertidor 1-FB-1. Las diferencias se deben principalmente a las pérdidas de conmutación, conducción, y cobre en el transformador con las del núcleo.

\section{APLICACIONES}

El Convertidor 1-FB-1 ofrece una alta versatilidad en aplicaciones de baja y media tensión, ya sea para disminuir o incrementar la fuente de entrada, proveyendo adicionalmente aislamiento galvánico.

A diferencia de los convertidores que pertenecen a la familia Boost, el Convertidor 1-FB-1 le permite al diseñador operar el convertidor como Buck para ciclos de trabajo cerca de uno; mientras que permite obtener altas tensiones, con ciclos de trabajo tendiendo a cero. Es definitivamente una gran ventaja que no se observa en otros convertidores tipo Boost.

El Convertidor 1-FB-1 se posiciona con características similares al convertidor Flyback, aunque éste último pertenece a la familia Buck-Boost. Desde el punto de vista de los costos de los semiconductores, se prevé sea similar para ambos convertidores, bajo la misma densidad de potencia, provisto que el Convertidor 1-FB ${ }^{-1}$ sea operado únicamente con la pareja Transistor-Diodo requerida para el punto de operación del ciclo de trabajo deseado.

\section{REFERENCIAS BIBILIOGRÁFICAS}

Anzicek, J. \& Thompson, M. (2005). DCDC boost converter design for Kettering Universitys GEM fuel cell vehicle. Proceedings on Electrical Insulation Conference and Electrical Manufacturing Expo, $307-316$.

Dubey, G. (1989). Power semiconductor controlled drives. Englewood Cliffs, NJ: Prentice Hall.

Erickson, R. \& Maksimovic, D. (2001). Fundamentals of power electronics. Norwell, MA: Kluwer Academic Publishers.

Fanghua, Z., Lan, X. \& Yangguang, Y. (2004). Bi-directional forward-flyback DC-DC converter. IEEE 35th Annual Power Electronics Specialists Conference, 5, 4058 - 4061.

François, C. (2006). Génie électrique. Bargue, Paris: Ellipses.

Gwyther, H. (1993). Potencia Eléctrica y Electrónica de Potencia. México DF, MX: Alfaomega.

Hart,D.(1997). Introduction to power electronics. Upper Saddle River, NJ: Prentice Hall.

Hirose, T., Nishimura, K. \& Kimura, T. (2010). An ac-link bidirectional DC-DC converter with synchronous rectifier. 36th Annual Conference on IEEE Industrial Electronics Society, 351 - 357.

Inoue, S. \& Akagi, H. (2007). A Bidirectional DC-DC Converter for an Energy Storage System With Galvanic Isolation. IEEE Transactions on Power Electronics, 22(6), 2299 - 2306. 
Kaiwei, Y., Yuancheng, R. \& Jia, W. (2003). A family of buck-type DC-DC converters with autotransformers. Eighteenth Annual IEEE Applied Power Electronics Conference and Exposition, 1, $114-120$.

Kassakian, J., Schlecht, M. \& Verghese, G. (1991). Principles of power electronics. Reading, Massachusetts: Addison-Wesley Publishing Company.

Kazimierczuk, M. \& Massarini, A. (1997). Feedforward control of DC-DC PWM boost converter. IEEE Transactions on Circuits and Systems I: Fundamental Theory and Applications, 44(2), 143-148.

Luo, F. \& Ye, H. (2004). Advanced DC/DC converters. Boca Raton, FL: CRC Press.

Middlebrook, R. \& Çuk, S. (1983). Advances in Switched-Mode Power Conversion. Pasadena, CA: TESLAco.

Mohan, N., Undeland, T. \& Robbins, W (2009). Electrónica de Potencia Convertidores. México DF, MX: Mc Graw Hill.

Rahid,M.(1995).Electrónicade potencia.Mexico, MX: Prentice Hall Hispanoamericana.

Seguí, S., Gimeno, F. \& Sánchez, C. (2004). Electrónica de Potencia. México DF, MX: Alfaomega.

Ray-Lee, L., Chi-Rung, P. \& Kuang-Hua, L. (2009). Family of single-inductor multioutput DC-DC converters. International Conference on Power Electronics and Drive Systems, 1216 - 1221.

Rosas-Caro, J.C., Ramirez, J.M. \& Peng, F.Z. (2010). A DC-DC multilevel boost converter.
IEEE Transactions on Power Electronics, 3(1), $129-137$.

Sato, E.K., Kinoshita, M. \& Sanada, K. (2010). Double DC-DC converter for uninterruptible power supply applications. IPEC Power Electronics Conference, 635 - 642.

Séguier, G., Bausière, R. \& Labrique, F. (2004). Électronique de pusissance. Dunod, Paris: Dunod. 8e édition.

Sukin, P. \& Yujin, S. (2011). An interleaved half-bridge bidirectional dc-dc converter for energy storage system applications. IEEE 8th International Conference on Power Electronics and ECCE Asia (ICPE \& ECCE), $2029-2034$.

Wei, S. \& Lehman, B. (2007). Current-Fed Dual-Bridge DC-DC Converter. IEEE Transactions on Power Electronics, 22(2), $461-469$.

Yamadaya, M., Owa, M. \& Matsuo, H. (2008). Small-size boost type DC-DC converter with the tiny embedded inductor. IEEE 30th International Telecommunications Energy Conference, 1 - 5 .

\section{SOBRE EL AUTOR}

Luis Alejandro Gólcher Barguil, Ingeniero Eléctrico. Máster en Ciencias de la Ingeniería Eléctrica. Profesor de la Escuela de Ingeniería Eléctrica de la Universidad de Costa Rica. Teléfono: +506 88379460 .

Correo electrónico: luis.golcher@gnecorp.com 
